\title{
A Lightweight First-Level Muon Track Trigger for Future Hadron Collider Experiments
}

\author{
D. Cieri*, S. Abovyan, V. Danielyan, M. Fras, P. Gadow, O. Kortner, S. Kortner, H. \\ Kroha, F. Müller, S. Nowak, P. Richter and K. Schmidt-Sommerfeld \\ Max-Planck-Institut für Physik, Föhringer Ring 6, 80805 Munich, Germany \\ E-mail: davide.cieriecern.ch
}

Single muon triggers are crucial for the physics programmes at hadron collider experiments. To keep the trigger rates reasonably low they must be highly selective.

Muon systems at LHC experiments and at future colliders use two muon chamber system for triggering: fast trigger chambers to identify the bunch crossing and provide a coarse momentum estimation, and slower precision chambers, for precise measurements of the muon trajectory.

A fast lightweight track finding algorithm, based on the Hough Transform and Linear Regression techniques, has been designed and implemented on a Zynq SoC device, reconstructing successfully muon tracks in a single trigger sector.

Topical Workshop on Electronics for Particle Physics (TWEPP2018)

17-21 September 2018

Antwerp, Belgium

${ }^{*}$ Speaker. 


\section{Introduction}

Highly selective first level muon triggers will still be crucial to undertake the demanding physics programme of future hadron collider experiments, like those at the High Luminosity LHC (HL-LHC) [1] or at the Future Circular Hadron Collider (FCC-hh) [2]. The high luminosities foreseen for these experiments place stringent limits on the first-level trigger rates. Therefore the future triggers must be able to discriminate between high- $p_{T}$ from low- $p_{T}$ muons.

This improvement in the muon $p_{T}$ resolution can be achieved in different ways. One possibility consists of using data from precise cylindrical drift tube detectors in the first-level trigger, as is planned for the upgraded ATLAS L0 Muon Trigger [3] at the HL- LHC and is proposed by the authors of this article for the FCC-hh baseline detector.

In this article we present a concept for a lightweight First-level Muon trigger based on the use of modern System-On-Chip technology. The presented system is based on a muon system geometry inspired by the ATLAS Muon Barrel spectrometer. The same concept is applicable to other geometries, like the proposed muon system of the baseline FCC detector.

\section{A lightweight first level muon track finder processor}

The considered muon system is composed of three chambers embedded in a strong magnetic field (Figure 1a). A muon chamber can be built by a joint use of fast gaseous detectors, like Resistive Plate Chambers (RPC), and precise gaseous detectors, such as muon drift tubes (DT). The transverse momentum of the muon particle can be calculated by measuring precisely the position and the direction of the muon track in the three chambers (track segments).

The proposed muon track trigger configuration makes use of both RPC and DT detectors to minimise the required processing power. On the other hand, the chosen architecture must also provide good trigger efficiency and have a low-latency (less than $1 \mu \mathrm{s}$ ) to satisfy usual trigger bandwidth requirements. The system therefore can be divided into two processing units (Figure 1b). The pre-trigger processes the information from the fast detectors to identify the bunch-crossings (BX) and the region-of-interests (RoIs) consistent with the current track candidate. Also a first coarse estimation of the track segment parameters can be realised.

A second stage consists of the Muon Track Finder Processor, which takes as input the drifttube hits that are matched with BX and RoIs identified by the pre-trigger and are utilised to precisely calculate the track segment parameters and, finally, the muon transverse momentum. The implemented segment finding algorithm is based on the use of a 1D Hough Transform [4].

\subsection{Segment finding with a 1D Hough Transform}

Muons with a relatively high transverse momentum (larger than $5 \mathrm{GeV} / \mathrm{c}$ ) are assumed to have a straight trajectory within a single drift tube chamber. For each hit of coordinates $(z, y)$, the following relation must be therefore valid,

$$
y=m z+b
$$

where $(m, b)$ are the track segment slope and intercept parameters. The distance between the hit and the drift tube anode wire is equal to the drift radius $r$, which is measured by the tube, 


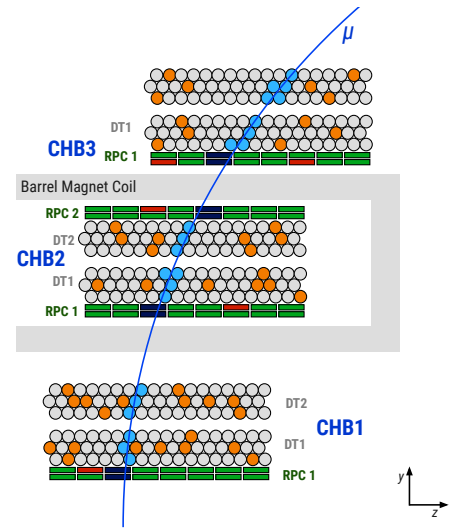

(a) The considered muon spectrometer geometry. The system is composed by three layers of DTs and RPCs immersed in a magnetic fields.

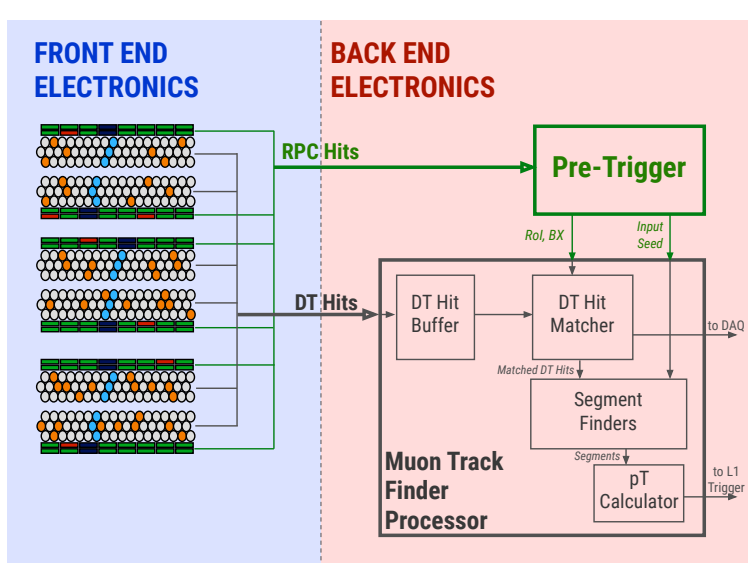

(b) Diagram of a possible readout chain for a muon trigger sector.

Figure 1

$$
r=\frac{\left|y_{t}-m z_{t}+b\right|}{\sqrt{1+m^{2}}}
$$

where $\left(z_{t}, y_{t}\right)$ are the coordinates of the tube wire. By inverting this relation it is possible to calculate for each hit the two possible values of the intercept,

$$
b_{ \pm}= \pm \sqrt{1+\bar{m}^{2}} \cdot r-\left(\bar{m} z_{t}-y_{t}\right),
$$

where $\bar{m}$ is the slope parameter estimate coming from the pre-trigger. To solve the two-fold ambiguity, a histogram of $b_{ \pm}$is filled with the DT hits where the maxima correspond to possible track candidates (Figure 2). Hits belonging to maxima are selected and used to compute the precise segment parameters by means of simple linear regression fitter.

\subsection{Transverse momentum calculation}

Once the segment fitting stage is completed, the final muon momentum calculation can take place. The muon transverse momentum is related to the curvature radius $R$ and to the magnetic field $B$ by the equation,

$$
p_{T}=B R .
$$

If only two of three segments have been reconstructed $R$ can be estimated by measuring the deflection angle $\Delta \theta$, which is the polar-angle difference between the segments in the two chambers,

$$
R=\frac{\Delta y}{2 \sin \Delta \theta},
$$

with $\Delta y$ the distance between the segments along the $y$ axis. If segments are reconstructed in all three chambers, the more precise sagitta method can be used [5].

$$
R=\frac{8 s}{(\Delta y)^{2}},
$$




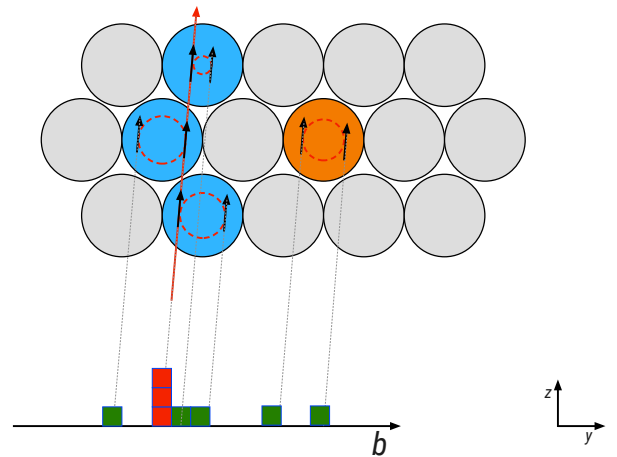

(a) The 1D Hough Transform method. Two values of $b_{ \pm}$are computed for each DT hit. A histogram of $b$ is then filled and segment candidates are identified by the bin with the largest hit content.

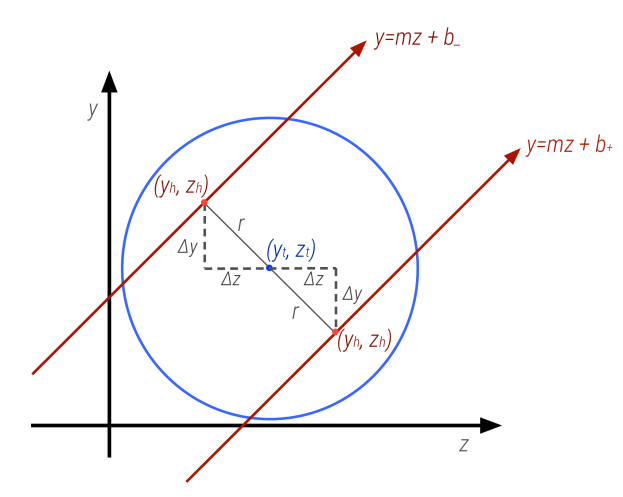

(b) Drift tube hit coordinates and their relation to the tube parameters.

Figure 2

where the sagitta $s$ is defined as the distance between the position of one segment from the straight line joining the remaining two. In the case of a non-homogeneous magnetic field, Equation 2.4 can be approximated by a series expansion dividing the detector volumes in small regions.

\section{Hardware implementation of the muon segment finding algorithm}

In order to demonstrate the feasibility of the proposed system and measure its performance, the muon track finding algorithm has been implemented in hardware on a System-on-Chip (SoC) Xilinx Zynq device ${ }^{1}$, mounted on a Xilinx ZC706 evaluation board [6].

The segment finder and the transverse momentum computation stages have been integrated in different parts of the device. The three segment finders (one per each muon chamber) have been implemented in the Programmable Logic (PL) to exploit the fast parallel processing of the FPGA architecture. The reconstructed segments are then transmitted via AXI bus to the Processing System (PS), where the precise floating point calculation of muon transverse momentum has been implemented.

The algorithm has been validated using our own generated single muon simulated samples. A software emulation of the algorithm has been also developed, implementing the same digitised equations as the firmware. A very good agreement between hardware and emulation has been observed. About $99.9 \%$ of the segments have been correctly reconstructed by both hardware and emulation.

The implemented firmware presents a low-latency, requiring about $400 \mathrm{~ns}$ to complete the whole process from the first hit coming in to the computation of the muon $p_{T}$ with a PL speed of $240 \mathrm{MHz}$.

\footnotetext{
${ }^{1}$ Xilinx xc7z045ffg900-2
} 
With the exception of needing about $93 \%$ of the available DSPs, the resource usage is relatively light (Table 1), requiring about one third of the logic cells of the chip. The large usage of DSPs is due to the arithmetical needs of the linear regression segment fitters.

Table 1: Resource utilisation of the full Muon Track Finder processor as implemented in the Xilinx Zynq XC7045 SoC device [6].

\begin{tabular}{ccccc}
\hline & LUTs $\left[10^{3}\right]$ & DSPs & FFs $\left[10^{3}\right]$ & BRAM (36 Kb) \\
\hline Muon Track Finder & 70.2 & 834 & 44.4 & 10.5 \\
Available in Zynq 7045 & 218 & 900 & 437 & 545 \\
\hline
\end{tabular}

\section{Conclusions}

A lightweight concept for a first level muon track finder for future hadron collider experiments has been presented. The proposed architecture is based on the combined use of fast and precise gaseous detectors, such as RPCs and cylindrical drift tubes. The system has been validated in simulation and hardware, using a hardware demonstrator based on commercially available technology. The performance of the system fulfils the typical needs of a first level muon trigger in terms of trigger efficiency and latency. The hardware implementation of the algorithm requires relatively few logic resources, making the architecture cost-friendly for applications in large hadron collider experiments.

\section{References}

[1] Burkhard Schmidt, The High-Luminosity upgrade of the LHC: Physics and Technology Challenges for the Accelerator and the Experiments, J. Phys. : Conf. Ser. 706.2 (2016), 022002. 42 p.

[2] M. Krammer, The update of the European strategy for particle physics, Phys. Scr. 14019 (2013)

[3] The ATLAS Collaboration, ATLAS Trigger and Data Acquisition Phase-II Upgrade Technical Design Report, Tech. Rep. (2017) CERN-LHCC-2017-020; ATLAS-TDR-029 https://cds.cern.ch/record/2285584.

[4] H.P.V. C. Method and means for recognizing complex patterns, US Patent 3,069,654. (1962)

[5] P. Gadow, Development of a Concept for the Muon Trigger of the ATLAS Detector at the HL-LHC, (2016) CERN-THESIS-2016-056

[6] Xilinx, Zynq-7000 All Programmable SoC Data Sheet: Overview (2017) 